Vol. 7, No. 5, Sep-0ct 1993, pp. 417-433

\title{
Learning Gravity Compensation in Robots: Rigid Arms, Elastic Joints, Flexible Links
}

\author{
ALESSANDRO DE LUCA AND STEFANO PANZIERI \\ Dipartimento di Informatica e Sistemistica \\ Università degli Studi di Roma "La Sapienza" \\ Via Eudossiana 18, 00184 Roma, Italy \\ Tel: +39644585-371 - Fax: +39644585-367 \\ E-mail: deluca@irmunisa.ing.uniroma1.it \\ panzieri@irmunisa.ing.uniroma1.it
}

\begin{abstract}
SUMMARY
The set-point regulation problem for robotic manipulators is a basic task that can be solved either by PID control or by model-based gravity compensation. These approaches are commonly applied both to rigid arms and to robots with flexible links and/or elastic joints. However, PID control requires fine and lengthy tuning of gains in order to achieve good performance on the whole workspace. Moreover, no global convergence proof has yet been given for this control law in case of flexible links or elastic joints. On the other hand, a constant or even a configuration-dependent gravity compensation is only an approximate solution when an unknown payload is present or when model parameters are poorly estimated. In this paper, a simple iterative scheme is proposed for generating exact gravity compensation at the desired set-point, without the knowledge of dynamic model terms. The resulting control law is shown to be global asymptotically stable for rigid arms as well as for manipulators with elastic joints or flexible links. Starting with a PD action on the error at the joint (viz. motor) level, an additional feedforward term is built and updated at discrete instants. Convergence of the scheme is proved under a mild condition on the proportional gain, related to a bound on the gravity terms. In the presence of concentrated or distributed flexibility, a structural property of the joint or of the link stiffness is further required, largely satisfied in practice. Simulation results are given for a three-link rigid arm while experimental results are also presented for a two-link robot with a flexible forearm.
\end{abstract}

KEY WORDS Robot Control, Regulation, Elastic Joints, Flexible Manipulators, Iterative Learning 


\section{INTRODUCTION}

Regulation of multi-link manipulators is often performed using linear feedback laws that exploit inherent physical properties of the mechanical system. In absence of gravity, it can be shown that a simple proportional derivative (PD) feedback at the joint (motor) error level is sufficient to asymptotically stabilize any arm configuration. This result is formally proved in [1] for rigid arms, in [2] for robots with joint elasticity, and in [3] when dealing with distributed link flexibility. In this latter case, a full state feedback including the deflection variables may improve the transient characteristics [4].

For rigid manipulators under gravity, the most direct approach to set-point regulation is to globally cancel the gravity terms and still apply PD control with positive definite gains. This leads to a nonlinear feedback control law which may be difficult to implement, but yields exponential stability. Under a mild condition on the proportional gain, this scheme can be simplified to constant gravity compensation, as evaluated at the desired configuration $[1,5]$; a purely linear feedback law with a feedforward action is then obtained (shortly denoted as PD+). In this case, the proportional gain should be chosen so to dominate the gradient of the gravity force in the whole robot workspace.

When flexible components are present in the robotic structure, a similar strategy based on PD plus a constant gravity feedforward has been shown to asymptotically stabilize also robots with elastic joints [2] and with flexible links [6]. For the elastic joint case, feedback is closed only around the motor variables, while for the flexible link case only the joint (rigid) variables are used for control. This control strategy works under a further structural assumption on the joint and on the link stiffness, respectively.

In all cases, an exact knowledge of the gravity vector is assumed. This is difficult to be achieved, e.g. for a robot picking up multiple unknown payloads, and therefore an on-line identification or an adaptive procedure would be required. In particular, the latter

asks for a proper factorization of the nonlinear gravity term [5] and is quite sensitive to unmodeled dynamics.

For robots with flexible components, it is well known that controlling the end-effector is quite a different problem from controlling the motion of the arm joints. In this case, the source of steady-state end-effector error under pure joint PD control is two-fold: first, a displacement is present at the motor level just as in the fully rigid case; second, a further displacement is introduced by the arm (joint and/or link) deflection. High-gain feedback will reduce but not eliminate the total error, exciting in fact higher order deformation modes and leading to longer transition times due to the poorly damped oscillations. 
On the other hand, to compensate for gravity effects, a standard model-independent remedy is the addition of an integral term to the linear PD law. However, several problems arise with the design of an efficient PID control, partly due to the nonlinear nature of the robot dynamics. Typically, saturation will occurr during large transient phases and reset or anti-windup procedures have to be devised when starting far from the final position [7].

From a theoretical point of view, asymptotic stability of robot PID control has been proved only for rigid arms. This result holds locally around the desired configuration and requires complex inequalities among the proportional, derivative, and integral gains to be satisfied $[8,9]$. In practice, some of these drawbacks may be overcome by adding the integral action only near the final point, so that gross motion is performed with PD control, while fine positioning is achieved with PID. However, no formal proof of convergence has been given for this modified law.

In this paper, we consider the set-point regulation problem under gravity for general manipulators, i.e. with or without joint elasticity and with or without link flexibility. A fast iterative scheme is proposed that builds up the required compensation at the final configuration, with a very limited knowledge about the robot gravity terms. A PD-based control law is applied iteratively at the motor level while a constant gravity feedforward is learned at discrete instants, without the explicit introduction of an integral error term nor the use of high-gain feedback.

An easy to check sufficient condition is derived that guarantees the global asymptotic convergence of the scheme to zero steady-state error, taking into account the robot nonlinearities in the analysis. This condition is obtained within a general modeling set-up, and then particularized for the two cases of fully rigid arms and of robots with elastic joints but rigid links. Whenever arm deflections are present, it is further assumed that arm stiffness dominates gravity effects, in analogy with [2] and [6]. This mild assumption is always satisfied in real robots.

Simulation results showing the performance of the overall scheme are presented for a three-link rigid arm moving in the vertical plane. Experimental results are also reported for the two-link lightweight manipulator, with a flexible forearm, available at the Robotics Laboratory of our Department [10]. This arm has been tilted from the horizontal plane so to include gravity effects.

\section{ROBOT MODEL PROPERTIES}

The dynamic model properties relevant for our control approach are reviewed here for rigid robots, for robots with rigid links but flexible joints, and for robots with flexible links. 
Consider first a robot arm composed of a serial chain of $n$ rigid links, actuated at the joints by motors connected through rigid transmissions. Denoting by $\theta$ and $u$ the $n \times 1$ vectors of motor coordinates and motor torques (both already scaled by the reduction ratio), the standard dynamic equations of motion are written as

$$
B(\theta) \ddot{\theta}+h(\theta, \dot{\theta})+g(\theta)=u
$$

with the $n \times n$ positive definite symmetric inertia matrix $B$, the $n \times 1$ vectors $h$ and $g=(\partial U / \partial \theta)^{T}$ of Coriolis and centrifugal forces and of gravity forces, respectively. It can be shown that the Hessian of the gravitational potential energy $U$ is bounded

$$
\left\|\frac{\partial g}{\partial \theta}\right\|=\left\|\frac{\partial^{2} U}{\partial \theta^{2}}\right\| \leq \alpha_{1}
$$

both for rotational and prismatic joints [5]. In the following we will use as norm of a matrix $A$ the spectral one, i.e. $\|A\|=\sqrt{\lambda_{\max }\left(A^{T} A\right)}\left(\|A\|=\lambda_{\max }(A)\right.$, for a symmetric matrix).

When the motion transmission elements are elastic, a further set of $n$ generalized coordinates has to be considered in order to fully describe the robot arm configuration. In [2], the complete modeling is carried out using the link position variables $\theta_{\ell}$ in addition to the motor variables $\theta$. The potential energy associated to the joint elasticity is then written as $U_{e}=\frac{1}{2}\left(\theta_{\ell}-\theta\right)^{T} K\left(\theta_{\ell}-\theta\right)$, where $K>0$ is the joint stiffness diagonal matrix. The $2 n$ second-order differential equations of motion become:

$$
\left[\begin{array}{cc}
B_{1}\left(\theta_{\ell}\right) & B_{2}\left(\theta_{\ell}\right) \\
B_{2}^{T}\left(\theta_{\ell}\right) & B_{3}
\end{array}\right]\left[\begin{array}{c}
\ddot{\theta}_{\ell} \\
\ddot{\theta}
\end{array}\right]+C\left(\theta, \theta_{\ell}, \dot{\theta}, \dot{\theta}_{\ell}\right)\left[\begin{array}{c}
\dot{\theta}_{\ell} \\
\dot{\theta}
\end{array}\right]+\left[\begin{array}{c}
g_{1}\left(\theta_{\ell}\right) \\
0
\end{array}\right]+\left[\begin{array}{cc}
K & -K \\
-K & K
\end{array}\right]\left[\begin{array}{c}
\theta_{\ell} \\
\theta
\end{array}\right]=\left[\begin{array}{l}
0 \\
u
\end{array}\right] .
$$

In the above partitioning, the motor torques perform work only on the associated motor variables while the gravity forces act only on the link side. Similarly to (2), one has

$$
\left\|\frac{\partial g_{1}}{\partial \theta_{\ell}}\right\| \leq \alpha_{1}
$$

being structurally $g_{1} \equiv g$ of the rigid case [2].

In order to preserve a unified notation, we introduce the $n \times 1$ deflection variables $\delta=\theta_{\ell}-\theta$ to be used in place of $\theta_{\ell}$. Applying this linear transformation leads to a model of robots with elastic joints in the form

$$
\begin{gathered}
{\left[\begin{array}{cc}
B_{1}(\theta+\delta)+\left[B_{2}+B_{2}^{T}\right](\theta+\delta)+B_{3} & B_{1}(\theta+\delta)+B_{2}^{T}(\theta+\delta) \\
B_{1}(\theta+\delta)+B_{2}(\theta+\delta) & B_{1}(\theta+\delta)
\end{array}\right]\left[\begin{array}{l}
\ddot{\theta} \\
\ddot{\delta}
\end{array}\right]} \\
+\left[\begin{array}{l}
h_{1}(\theta, \delta, \dot{\theta}, \dot{\delta}) \\
h_{2}(\theta, \delta, \dot{\theta}, \dot{\delta})
\end{array}\right]+\left[\begin{array}{l}
g_{1}(\theta+\delta) \\
g_{1}(\theta+\delta)
\end{array}\right]+\left[\begin{array}{c}
0 \\
K \delta
\end{array}\right]=\left[\begin{array}{l}
u \\
0
\end{array}\right] .
\end{gathered}
$$


When considering the presence of flexible links, the Lagrangian technique can still be used to derive the dynamic equations of motion [11], using a finite set of space functions for describing link deformation shapes. A linear model is in general sufficient to capture the dynamics of each flexible link, with the slender links seen as Euler-Bernoulli beams with proper boundary conditions. By extending $\delta$ to be the $m \times 1$ coordinate vector of generalized deformations (possibly including both joint and link ones), the closed-form dynamic equations can be written as $n+m$ second-order nonlinear differential equations in the general form [12]:

$$
\left[\begin{array}{cc}
B_{\theta \theta}(\theta, \delta) & B_{\theta \delta}(\theta, \delta) \\
B_{\theta \delta}^{T}(\theta, \delta) & B_{\delta \delta}(\theta, \delta)
\end{array}\right]\left[\begin{array}{l}
\ddot{\theta} \\
\ddot{\delta}
\end{array}\right]+\left[\begin{array}{c}
h_{\theta}(\theta, \delta, \dot{\theta}, \dot{\delta}) \\
h_{\delta}(\theta, \delta, \dot{\theta}, \dot{\delta})
\end{array}\right]+\left[\begin{array}{c}
g_{\theta}(\theta, \delta) \\
g_{\delta}(\theta, \delta)
\end{array}\right]+\left[\begin{array}{c}
0 \\
K \delta
\end{array}\right]=\left[\begin{array}{l}
u \\
0
\end{array}\right] .
$$

Note that the link deformation is implicitly described in a frame clamped at the joint side, implying that the control does not enter directly in the equations of the flexible part.

Comparing (6) with (5), we immediately see that the joint elastic model is a particularization of the general flexible case, though with different terms. For our purposes, the most relevant difference is in the structure of the gravity vector $g=\left(g_{\theta}, g_{\delta}\right)$. In the elastic joint case, the two subvectors are equal $\left(g_{\theta}=g_{\delta}=g_{1}\right)$ and have a functional dependence limited to the sum $\theta+\delta$. This allows to bound the gradient of the whole gravity vector in (5), just based on inequality (4).

On the other hand, the more general dependence of the gravity components in (6) requires further analysis. In the hypothesis of small link deformation, model validity extends up to a limited amount of stored elastic potential energy: $U_{e}=\frac{1}{2} \delta^{T} K \delta \leq U_{e, \max }$, where the diagonal positive definite matrix $K$ is the one appearing in (6). As a result, denoting by $q=(\theta, \delta)$ the $(n+m) \times 1$ vector characterizing the arm configuration, we have the following inequality:

$$
\left\|\frac{\partial g}{\partial q}\right\| \leq \gamma_{0}+\gamma_{1}\|\delta\| \leq \gamma_{0}+\gamma_{1} \sqrt{\frac{2 U_{e, \max }}{\lambda_{\max }(K)}}=: \alpha
$$

where $\gamma_{0}, \gamma_{1}, \alpha>0$. Similarly,

$$
\left\|\frac{\partial g_{\theta}}{\partial q}\right\| \leq \alpha_{\theta}, \quad\left\|\frac{\partial g_{\delta}}{\partial q}\right\| \leq \alpha_{\delta}
$$

These can be easily proved by observing that the gravity terms contain only trigonometric functions of $\theta$ and linear/trigonometric functions of $\delta$. As a direct consequence of (7), we have

$$
\left\|g\left(q_{1}\right)-g\left(q_{2}\right)\right\| \leq \alpha\left\|q_{1}-q_{2}\right\|, \quad \forall q_{1}, q_{2} \in \mathbb{R}^{n+m}
$$


while similar inequalities are obtained from (8).

In the following, we will consider the general flexible model (6) but it is intended that the obtained results fully apply also to robots having rigid links, with proper simplifications. In particular, $\delta$ vanishes (together with $g_{\delta}$ ) in the rigid joint case so that $g=g_{\theta}$ and

$$
\alpha=\alpha_{\theta}=\alpha_{1}, \quad \alpha_{\delta}=0
$$

Instead, in the elastic joint case $g_{\theta}=g_{\delta}=g_{1}$ and

$$
\alpha=2 \alpha_{1}, \quad \alpha_{\theta}=\alpha_{\delta}=\alpha_{1},
$$

where the properties of the spectral norm of a matrix [13] have been taken into account.

\section{3. $\mathrm{PD}+\mathrm{CONTROL}$}

For a desired constant motor position $\theta_{d}$, under perfect knowledge of the gravity term, the input torque $u$ can be chosen as a linear $P D+$ control, i.e.

$$
u=K_{P}\left(\theta_{d}-\theta\right)-K_{D} \dot{\theta}+g_{\theta}\left(\theta_{d}, \delta_{d}\right), \quad K_{P}>0, K_{D}>0
$$

being the associated $\delta_{d}$ implicitly defined as the solution of

$$
g_{\delta}\left(\theta_{d}, \delta\right)+K \delta=0
$$

This includes the case of fully rigid arms [1], where $\delta_{d} \equiv 0$, and that of elastic joints [2], where $\delta_{d} \neq 0$.

For the dynamic model (6) it has been shown in [6] that, under the assumption

$$
\lambda_{\min }\left(\begin{array}{cc}
K_{P} & O \\
O & K
\end{array}\right)>\alpha
$$

the state $q=q_{d}=\left(\theta_{d}, \delta_{d}\right), \dot{q}=0$ is the unique equilibrium of the closed-loop system $(6,10)$, i.e. satisfying

$$
\begin{aligned}
& g_{\theta}(\theta, \delta)=K_{P}\left(\theta_{d}-\theta\right)+g_{\theta}\left(\theta_{d}, \delta_{d}\right) \\
& g_{\delta}(\theta, \delta)=-K \delta
\end{aligned}
$$

Condition (14) can always be satisfied, provided that an assumption on the structural flexibility holds:

$$
\lambda_{\min }(K)=\min _{i=1, \ldots, n}\left\{k_{i}\right\}>\alpha
$$


being $K$ diagonal. This is not restrictive in general, and depends on the relative magnitude of link and/or joint stiffness vs. gravity. As a result, by choosing the proportional control gain so that $\lambda_{\min }\left(K_{P}\right)>\alpha$, the equilibrium state $q=q_{d}, \dot{q}=0$ of system (6) under control (12) is asymptotically stable.

We finally remark that the same condition (14) guarantees an unique equilibrium point also when an approximate constant gravity compensation $\widehat{g}_{\theta}$ is used in (12) in place of $g_{\theta}\left(\theta_{d}, \delta_{d}\right)$. In this case, the equilibrium point will indeed be a $\bar{q}=(\bar{\theta}, \bar{\delta}) \neq q_{d}$.

\section{ITERATIVE CONTROL SCHEME}

An iterative compensation scheme that achieves set-point regulation in the general (flexible) robot without knowledge of gravity is introduced as follows. In particular, our objective will be to drive the vector of robot motor variables $\theta$ to a specified value $\theta_{d}$.

Let $q_{0}=\left(\theta_{0}, \delta_{0}\right)$ be the initial arm configuration. The control law during iteration $i$ is defined as

$$
u=\frac{1}{\beta} K_{P}\left(\theta_{d}-\theta\right)-K_{D} \dot{\theta}+u_{i-1}, \quad \beta>0
$$

for $i=1,2, \ldots$, where the term $u_{i-1}$ is a constant feedforward. If $u_{0}=0$, which is the common initialization, the first iteration is performed with a simple joint PD control. Indeed, one may collect the best available information on the required gravity term by setting $u_{0}=\widehat{g}_{\theta}\left(q_{d}\right)$, where the 'hat' denotes an estimate.

At the end of the $i$ th iteration, system (6) under control (18) reaches an equilibrium state $q=q_{i}=\left(\theta_{i}, \delta_{i}\right), \dot{q}=0$, satisfying

$$
\begin{aligned}
g_{\theta}\left(\theta_{i}, \delta_{i}\right) & =\frac{1}{\beta} K_{P}\left(\theta_{d}-\theta_{i}\right)+u_{i-1}, \\
g_{\delta}\left(\theta_{i}, \delta_{i}\right) & =-K \delta_{i} .
\end{aligned}
$$

Note that the unknown gravity term $g_{\theta}\left(q_{i}\right)$ is determined through the reading of the control effort at steady state. For the next iteration, the feedforward is istantaneously updated as

$$
u_{i}=\frac{1}{\beta} K_{P}\left(\theta_{d}-\theta_{i}\right)+u_{i-1}
$$

and control (18) is applied again, starting from the current configuration $q_{i}$. A block diagram of the overall controller is shown in Fig. 1. 


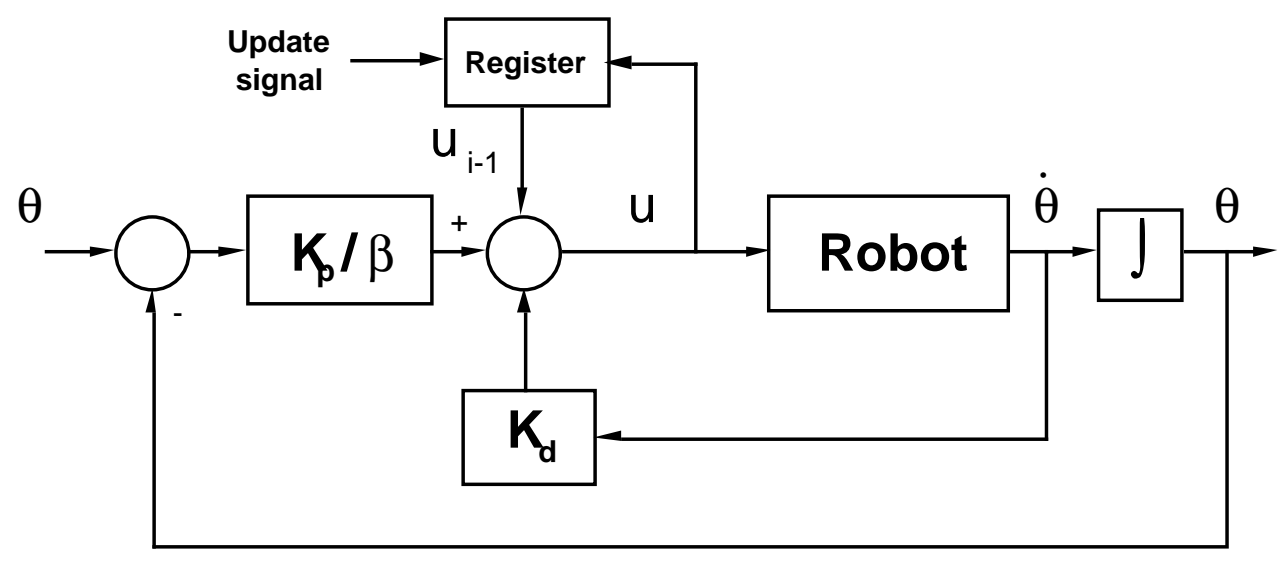

Fig. 1 - The iterative learning controller

Our main result is the following:

Theorem. The sequence $\left\{\theta_{0}, \theta_{1}, \ldots\right\}$ converges to $\theta_{d}$, starting from any initial $q_{0}$, provided that:

(a) $\quad \lambda_{\min }(K)>\alpha$;

(b) $\quad \lambda_{\min }\left(K_{P}\right)>\alpha$;

(c) $0<\beta \leq \frac{1}{2} \frac{\alpha-\alpha_{\delta}}{\alpha_{\theta}}$.

Proof. Let $e_{i}=\theta_{d}-\theta_{i}$. At the end of the $i$ th iteration, eqs. (19) and (21) imply that $u_{i}=g_{\theta}\left(q_{i}\right)$ at the steady state $q_{i}$, and so

$$
\begin{aligned}
\left\|u_{i}-u_{i-1}\right\| & =\left\|g_{\theta}\left(q_{i}\right)-g_{\theta}\left(q_{i-1}\right)\right\| \leq \alpha_{\theta}\left\|q_{i}-q_{i-1}\right\| \\
& \leq \alpha_{\theta}\left(\left\|\theta_{i}-\theta_{i-1}\right\|+\left\|\delta_{i}-\delta_{i-1}\right\|\right)
\end{aligned}
$$

where the first inequality (8) was used. From eq. (20), using the second inequality in (8) and hypothesis (a), we have

$$
\begin{aligned}
\left\|\delta_{i}-\delta_{i-1}\right\| & \leq\left\|K^{-1}\right\| \cdot\left\|g_{\delta}\left(q_{i}\right)-g_{\delta}\left(q_{i-1}\right)\right\|<\frac{1}{\alpha} \alpha_{\delta}\left\|q_{i}-q_{i-1}\right\| \\
& \leq \frac{\alpha_{\delta}}{\alpha}\left(\left\|\theta_{i}-\theta_{i-1}\right\|+\left\|\delta_{i}-\delta_{i-1}\right\|\right)
\end{aligned}
$$

or, since $\alpha>\alpha_{\delta}$,

$$
\left\|\delta_{i}-\delta_{i-1}\right\|<\frac{\alpha_{\delta}}{\alpha-\alpha_{\delta}}\left\|\theta_{i}-\theta_{i-1}\right\|
$$

Combining (22) and (24),

$$
\left\|u_{i}-u_{i-1}\right\|<\frac{\alpha \alpha_{\theta}}{\alpha-\alpha_{\delta}}\left\|\theta_{i}-\theta_{i-1}\right\| \leq \frac{\alpha \alpha_{\theta}}{\alpha-\alpha_{\delta}}\left(\left\|e_{i}\right\|+\left\|e_{i-1}\right\|\right) .
$$


On the other hand, from eq. (21)

$$
\left\|u_{i}-u_{i-1}\right\|=\frac{1}{\beta}\left\|K_{P} e_{i}\right\|
$$

From eqs. $(25,26)$, using hypothesis (b), it follows immediately

$$
\frac{1}{\beta} \alpha\left\|e_{i}\right\|<\frac{1}{\beta} \lambda_{\min }\left(K_{P}\right)\left\|e_{i}\right\| \leq \frac{1}{\beta}\left\|K_{P} e_{i}\right\|<\frac{\alpha \alpha_{\theta}}{\alpha-\alpha_{\delta}}\left(\left\|e_{i}\right\|+\left\|e_{i-1}\right\|\right) .
$$

Reorganizing terms, since hypothesis (c) implies $\alpha-\alpha_{\delta}-\beta \alpha_{\theta}>0$, we obtain

$$
\left\|e_{i}\right\|<\frac{\beta \alpha_{\theta}}{\alpha-\alpha_{\delta}-\beta \alpha_{\theta}}\left\|e_{i-1}\right\|
$$

Therefore, the error norm in (28) satifies a contraction mapping condition if

$$
\frac{\beta \alpha_{\theta}}{\alpha-\alpha_{\delta}-\beta \alpha_{\theta}} \leq 1
$$

which is again guaranteed by hypothesis (c). As a result, $\lim _{i \rightarrow \infty}\left\|e_{i}\right\|=0$, and asymptotic convergence of $\left\{\theta_{i}\right\}$ to $\theta_{d}$ is proved for any initial arm configuration $q_{0}$.

Q.E.D.

Hypothesis (b) for rigid robots and hypotheses (a) together with (b) for flexible ones are those needed to show that the motor PD control law with constant gravity compensation is globally asymptotically stable, when the gravity vector is known. In the present case, they are needed to ensure that the robot arm under control (18) has a unique steadystate solution at every iteration, thus enforcing safe operation against drifting motions outside $2 \pi$ rotations [1]. The new hypothesis (c) guarantees the convergence of the iterative scheme (21), and in particular that $\lim _{i \rightarrow \infty} u_{i}=g_{\theta}\left(q_{d}\right)$, with a priori knowledge limited to the bounds $(7,8)$ on the gravity terms.

A series of remarks are now in order:

- The same proof can be followed for rigid robot arms. Using (10), it follows from (c) that $\beta \leq \frac{1}{2}$, as first shown in [14]. Thus, merging conditions (b) and (c), the overall proportional gain matrix $\widehat{K}_{P}=K_{P} / \beta$ in (18) has to be chosen so to satisfy

$$
\lambda_{\min }\left(\widehat{K}_{P}\right)>2 \alpha=2 \alpha_{1}
$$

- In the case of elasticity concentrated only at the joint, using (11), it follows that $\beta \leq \frac{2 \alpha_{1}-\alpha_{1}}{2 \alpha_{1}}=1 / 2$, as in the rigid case. 
- For robots with flexible links, it is shown in [15] that when the lower part of the gravity vector can be assumed to be independent from $\delta$, i.e. $g_{\delta}=g_{\delta}(\theta)$, then a different bound can be derived for the gain $\beta$ as

$$
\beta \leq \frac{1}{2} \frac{\alpha}{\alpha_{\theta}\left(1+\frac{\alpha_{\delta}}{\alpha}\right)}
$$

which is strictly larger than the one given by condition (c), so that a smaller $\widehat{K}_{P}$ can be used.

- The iterative scheme can be interpreted as a discrete-time PID, in which the integral term is updated only at fixed instants. Moreover, this approach combines in an automatic way the benefits of a PD control far from the destination and of an integral action close to the goal, avoiding wind-up effects. As a further merit of the scheme, one should consider that gains with guaranteed global convergence properties are easily selected.

- The iterative scheme $(18,21)$ is also reminiscent of learning control algorithms that achieve reproduction of repetitive trajectories for rigid [16,17] or flexible robot arms $[18,19]$. However, no repositioning of the arm to the initial configuration is performed (nor required) here, at any iteration.

- The bounds $(7,8)$ on the gravity terms may be evaluated taking into account the maximum admissible payload, so to ensure exact set-point regulation in all operating conditions. Moreover, they can be directly obtained through experimental trials.

- As a drawback, since each update of the feedforward term should be performed at steady state, in principle the control scheme converges to the desired position in a doubly infinite time. However, ultimate boundedness of the error in finite time is obtained by updating the feedforward term as soon as error variations definitely drop below a given threshold, even before a complete stop. Moreover, it should be emphasized that a fixed PD control with gravity compensation guarantees not just asymptotic but rather exponential convergence to the unique equilibrium, as follows easily from the results in [20]. Therefore, for the present application a time bound can be derived in order to predict convenient updating instants.

- An interesting aspect is to estimate the distance from necessity of the sufficient conditions $(\mathrm{a}-\mathrm{c})$. This point can be investigated through simulations and experiments. In our experience, the above criteria are rather stringent as will be shown in the following sections. 


\section{SIMULATION RESULTS FOR A RIGID ROBOT}

The proposed control scheme has been simulated on a $3 \mathrm{R}$ rigid robot arm moving in the vertical plane (Fig. 2). The three robot links have equal length $\ell_{i}=0.5 \mathrm{~m}$, with uniformly distributed masses of 30,20 , and $10 \mathrm{~kg}$, respectively.

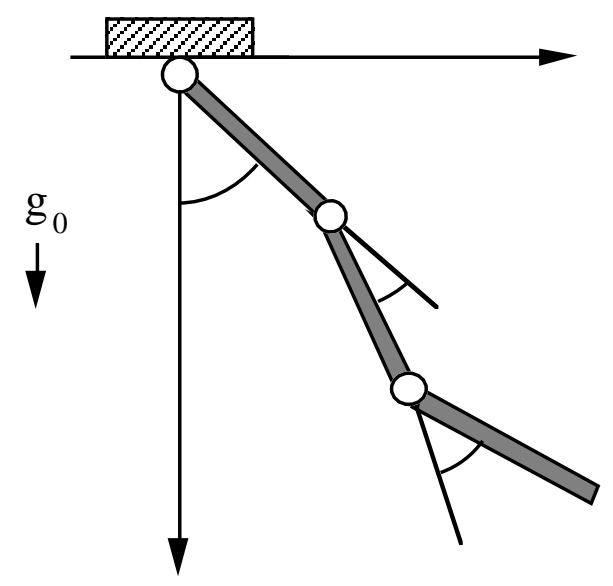

Fig. 2 - A $3 \mathrm{R}$ rigid robot arm in the vertical plane

In this case, the gravity vector is given by

$$
\begin{aligned}
g_{1}(\theta)= & g_{0}\left[\left(m_{1} d_{1}+m_{2} \ell_{1}+m_{3} \ell_{1}\right) \sin \theta_{1}+\left(m_{2} d_{2}+m_{3} \ell_{2}\right) \sin \left(\theta_{1}+\theta_{2}\right)\right. \\
& \left.+m_{3} d_{3} \sin \left(\theta_{1}+\theta_{2}+\theta_{3}\right)\right] \\
g_{2}(\theta)= & g_{0}\left[\left(m_{2} d_{2}+m_{3} \ell_{2}\right) \sin \left(\theta_{1}+\theta_{2}\right)+m_{3} d_{3} \sin \left(\theta_{1}+\theta_{2}+\theta_{3}\right)\right] \\
g_{3}(\theta)= & g_{0} m_{3} d_{3} \sin \left(\theta_{1}+\theta_{2}+\theta_{3}\right),
\end{aligned}
$$

where $d_{i}=\ell_{i} / 2, g_{0}=9.8$ is the gravity acceleration, and $\theta=(0,0,0)$ corresponds to the straight downward position (of minimum potential energy). With the nominal data of this rigid case, the maximum value $\alpha=\alpha_{1} \simeq 400$ is attained at $\theta=0$.

A motion from $\theta=0$ to the straight arm position of maximum gravity force $(\pi / 2$ of first joint rotation) is commanded, using as proportional and derivative gains

$$
\widehat{K}_{P}=\operatorname{diag}\{1000,600,280\}, \quad K_{D}=\operatorname{diag}\{200,100,20\}
$$

The update $(21)$ for $u_{i}$ is made at fixed intervals of 3 seconds. Figures $3-5$ and $6-8$ show the errors and the applied torques over 10 seconds. In this case, two updates are sufficient for regulating the error to zero within 7.5 seconds. Note that only the first position gain in (33) does satisfy the sufficient condition (30), while the gain of the third joint is even smaller than $\alpha_{1}$. 


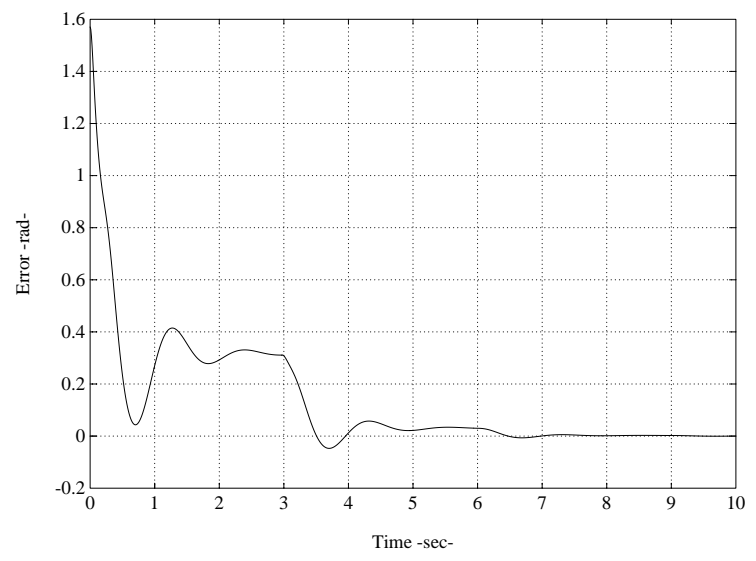

Fig. 3 - Position error for joint $1-q_{d}=(\pi / 2,0,0)$

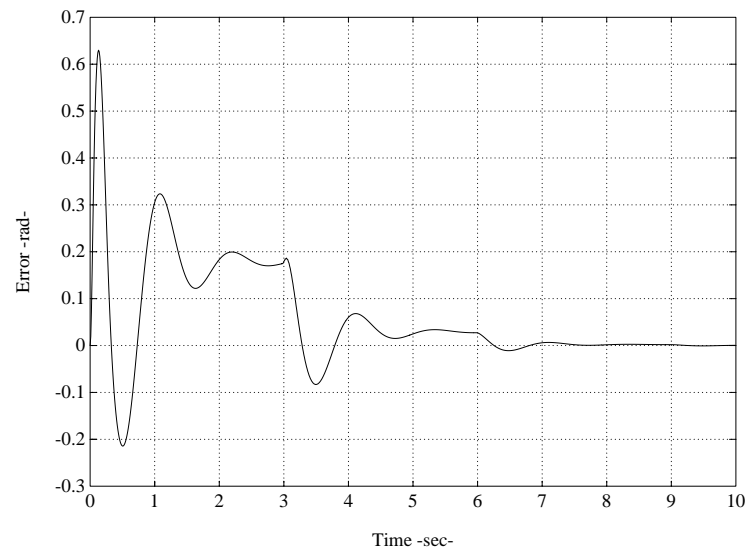

Fig. 4 - Position error for joint 2

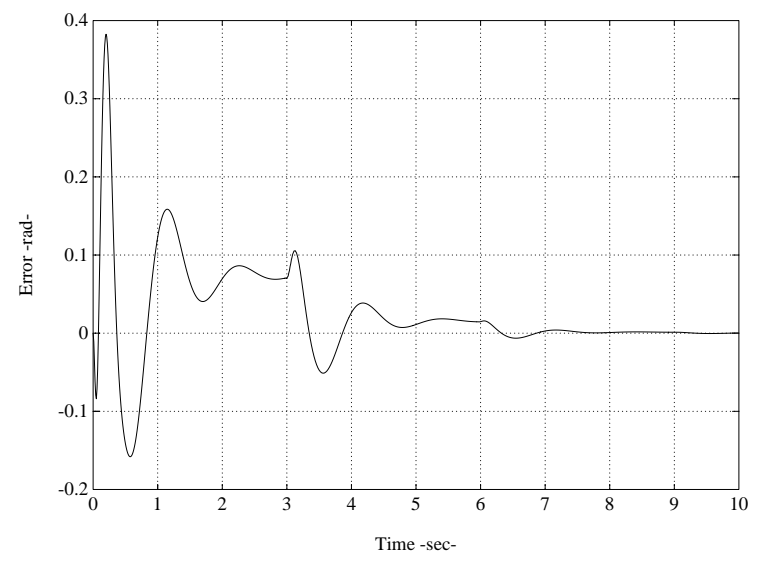

Fig. 5 - Position error for joint 3 


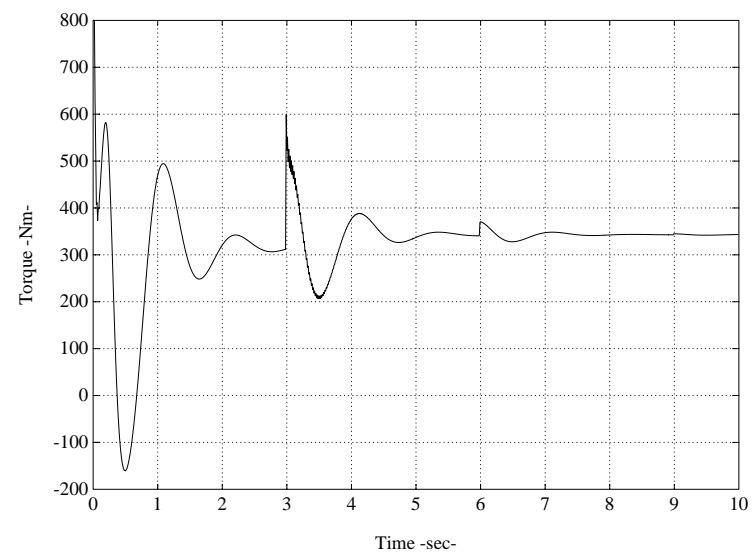

Fig. 6 - Applied torque for joint $1-q_{d}=(\pi / 2,0,0)$

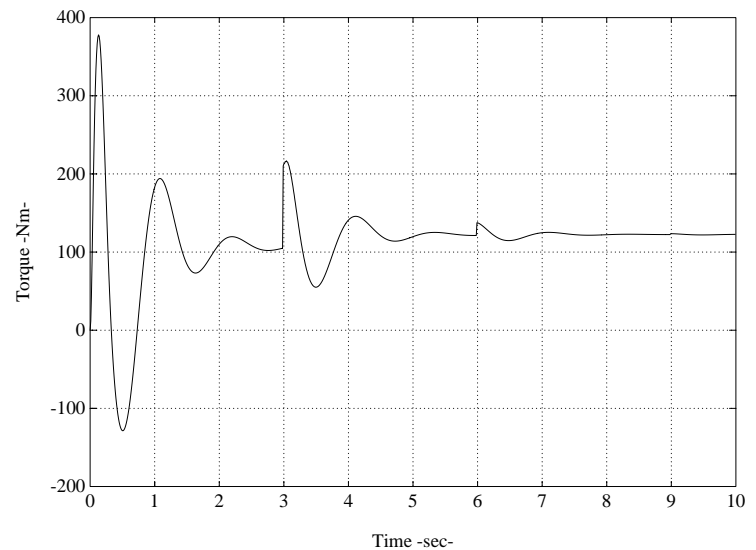

Fig. 7 - Applied torque for joint 2

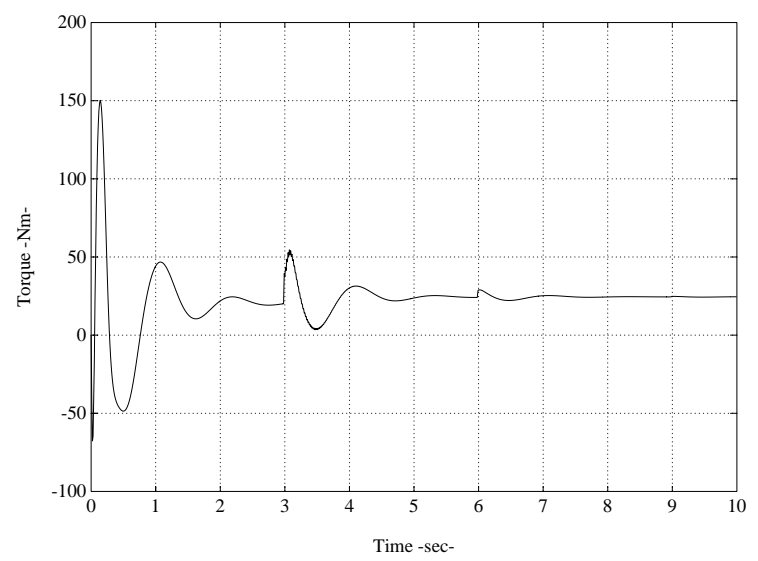

Fig. 8 - Applied torque for joint 3

As a second example, a regulation to $\theta_{d}=(3 \pi / 4,0,0)$ was attempted with smaller positional gains. In particular, $\widehat{K}_{P}=500 \cdot I$ was used, which satisfies condition (b) but not 
the bound (30). Update intervals for $u_{i}$ are slowed down to 6 seconds, in order to enable the reaching of steady-state conditions.

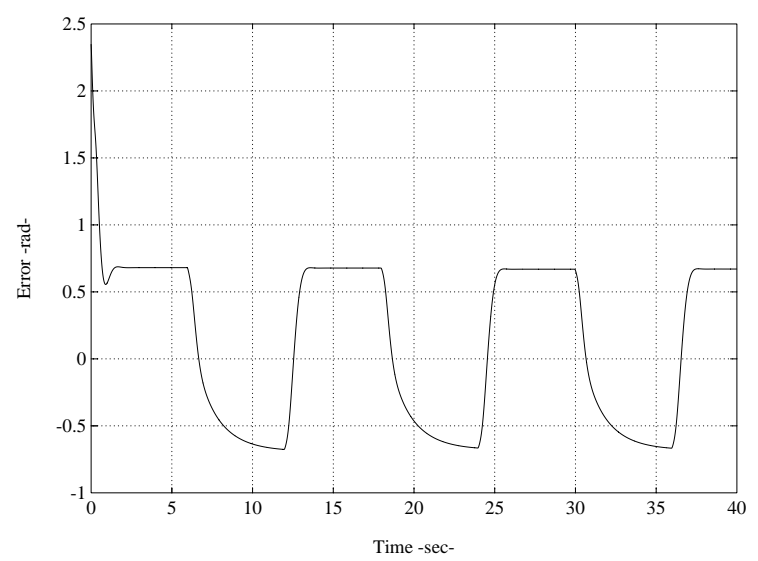

Fig. 9 - Position error for joint 1 with reduced gains $-q_{d}=(3 \pi / 4,0,0)$

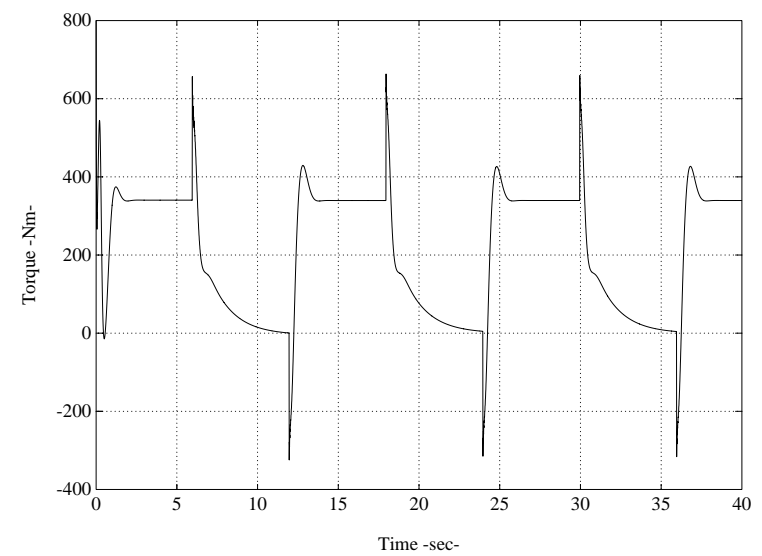

Fig. 10 - Applied torque for joint 1 with reduced gains

Figure 9 shows 40 seconds of the first joint position error. A persistent oscillatory behavior results as a consequence of the poor learning capabilities: the robot arm switches alternatively from an almost horizontal configuration, where the maximum torque effort is stored, to the upward straight configuration, where the error feedback torque counterbalances the learned feedforward term so to give a rather small net torque (see Fig. 10). Note also that, being $\widehat{\lambda}_{\min }\left(K_{P}\right)>\alpha_{1}$, there is still a unique equilibrium configuration for each applied feedforward. As a result, this choice of reduced gains gives a quantitative information on how much the sufficient condition (30) could be relaxed in rigid robot arms.

\section{EXPERIMENTAL RESULTS FOR A FLEXIBLE ROBOT}

The design of the iterative control algorithm has been carried out also for the two-link 
lightweight manipulator with a flexible forearm available at the Robotics Laboratory of our Department. The arm is a planar mechanism constituted by two links, respectively $\ell_{1}=0.3 \mathrm{~m}$ and $\ell_{2}=0.7 \mathrm{~m}$ long, connected by revolute joints, with direct-drive actuators, and mounted on a fixed basement as shown in Fig. 11.

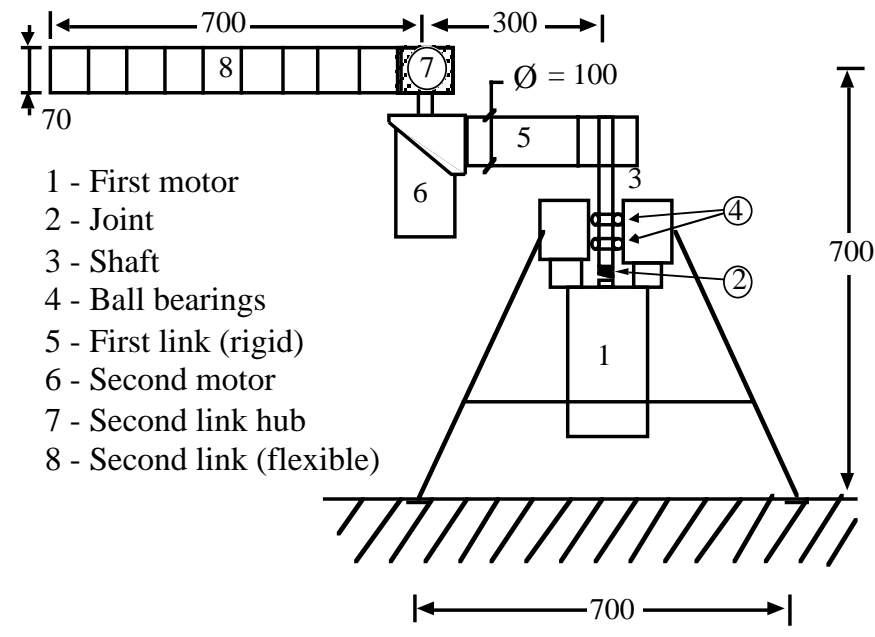

Fig. 11 - The two-link flexible robot arm at DIS

The upper link is rigid while the second link, weighting $m_{2}=1.8 \mathrm{~kg}$, is very flexible in the plane of motion. The first two natural frequencies of vibration are

$$
f_{1}=4.716 \mathrm{~Hz}, \quad f_{2}=14.395 \mathrm{~Hz}
$$

with stiffness coefficients of the diagonal matrix $K$ being

$$
k_{1}=878.02 \mathrm{~N}, \quad k_{2}=8180.56 \mathrm{~N}
$$

A Lagrangian dynamic model of this flexible robot can be found in [10]. In our experiments, the manipulator base has been tilted by $\gamma_{t} \simeq 6^{\circ}$ from the horizontal plane, so to include gravity effects. The associated model term $g(\theta, \delta)$ is reported below, including only the first two modes:

$$
g_{\theta}=\left[\begin{array}{l}
g_{1} \\
g_{2}
\end{array}\right]^{\mathrm{T}}, \quad g_{\delta}=\left[\begin{array}{l}
g_{3} \\
g_{4}
\end{array}\right]^{\mathrm{T}}
$$

with

$$
\begin{aligned}
& g_{1}=A_{1} \sin \theta_{1}+A_{2} \sin \left(\theta_{1}+\theta_{2}\right)+\left(A_{3} \delta_{1}+A_{4} \delta_{2}\right) \cos \left(\theta_{1}+\theta_{2}\right) \\
& g_{2}=A_{2} \sin \left(\theta_{1}+\theta_{2}\right)+\left(A_{3} \delta_{1}+A_{4} \delta_{2}\right) \cos \left(\theta_{1}+\theta_{2}\right) \\
& g_{3}=A_{3} \sin \left(\theta_{1}+\theta_{2}\right) \\
& g_{4}=A_{4} \sin \left(\theta_{1}+\theta_{2}\right) .
\end{aligned}
$$


The constant coefficients are:

$$
\begin{aligned}
& A_{1}=g_{0}\left(m_{1} \ell_{c 1}+\left(m_{2}+m_{h 2}+m_{p}\right) \ell_{1}\right) \\
& A_{2}=g_{0}\left(m_{2} \ell_{c 2}+m_{p} \ell_{2}\right) \\
& A_{3}=g_{0}\left(m_{p} \phi_{1 e}+v_{1}\right) \\
& A_{4}=g_{0}\left(m_{p} \phi_{2 e}+v_{2}\right),
\end{aligned}
$$

being $g_{0}=9.8 \sin \gamma_{t}$ the gravity acceleration, and with $m_{1} \ell_{c 1}=0.111 \mathrm{~kg} \cdot \mathrm{m}, m_{2} \ell_{c 1}=$ $0.537 \mathrm{~kg} \cdot \mathrm{m}, m_{h 2}=3.1 \mathrm{~kg}, m_{p}=0 \mathrm{~kg}, \phi_{1 e}=-1.446 \mathrm{~m}, \phi_{2 e}=1.369 \mathrm{~m}, v_{1}=0.48 \mathrm{~kg} \cdot \mathrm{m}$, $v_{2}=0.18 \mathrm{~kg} \cdot \mathrm{m}$.

With the used convention, $q=\left(\theta_{1}, \theta_{2}, \delta_{1}, \delta_{2}\right)=(0,0,0,0)$ corresponds to the straight downward position. Note that in this robot $g_{\delta}$ is only a function of $\theta$, so that the bound (31) can be used for the gain $\beta$. A value $\alpha \simeq 2.85$ is obtained for the nominal data, while the same value is used as an upper limit for $\alpha_{\theta}$ and $\alpha_{\delta}$. From (35), it is also apparent that the structural hypothesis (a) is largely satisfied.

In the first experiment, a motion from $q=0$ (undeformed arm) to the straight position of maximum gravity force ( $\pi / 2$ of first joint clockwise rotation) is commanded, using as proportional and derivative gains

$$
\widehat{K}_{P}=\operatorname{diag}\{10.7,11.6\}, \quad K_{D}=\operatorname{diag}\{1.6,0.85\} .
$$

The update (21) for $u_{i}$ is made at fixed intervals of 5 seconds. Figures 12-15 show the joint errors and the applied torques over 14 seconds. Two updates are sufficient for regulating the error to zero within 11 seconds, since the position gains in (39) satisfy the combined condition (b) and (c).

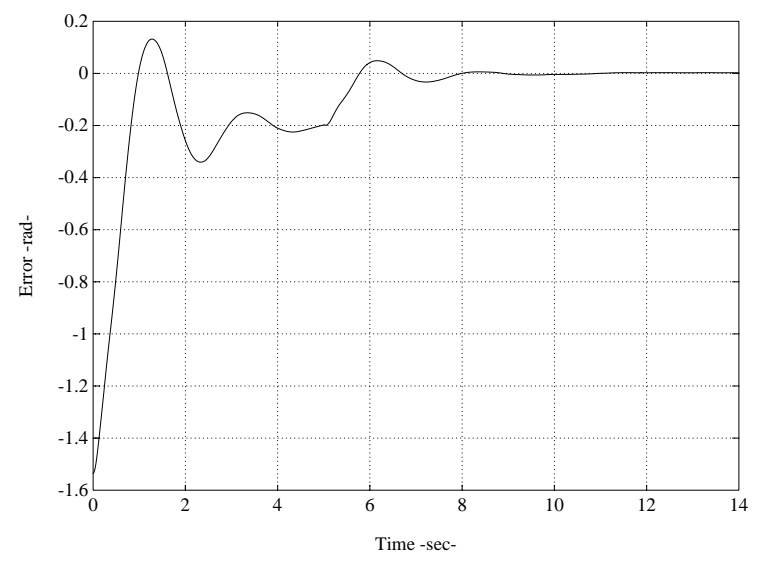

Fig. 12 - Position error for joint $1\left(\theta_{d}=(-\pi / 2,0)\right)$ 


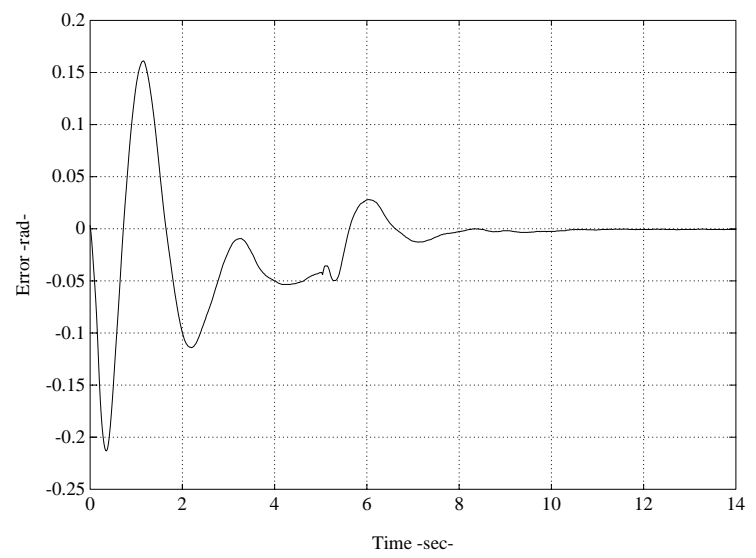

Fig. 13 - Position error for joint 2

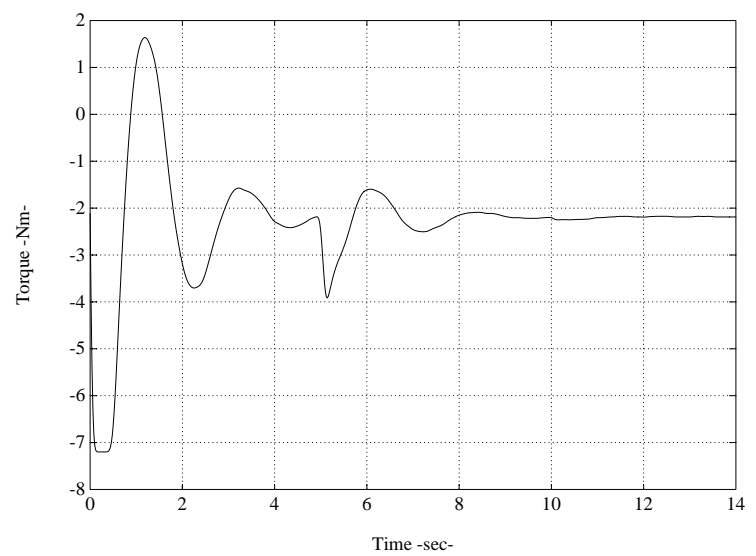

Fig. 14 - Applied torque for joint 1

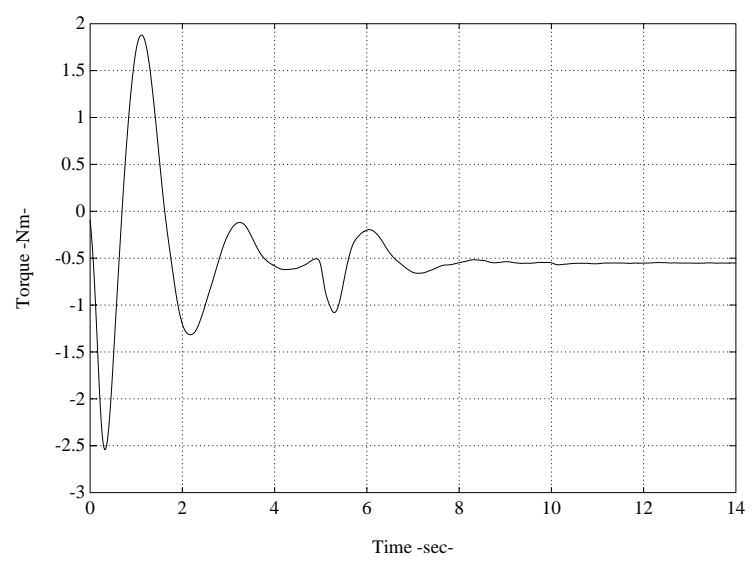

Fig. 15 - Applied torque for joint 2

The evolution of the tip deflection angle, as seen from the second link base, is given in Fig. 16, indicating that a maximum deflection of $\approx 0.7 \cdot 9 \cdot(\pi / 180)=10 \mathrm{~cm}$ is attained during motion while the residual tip deformation is $\approx 2 \mathrm{~cm}$. 


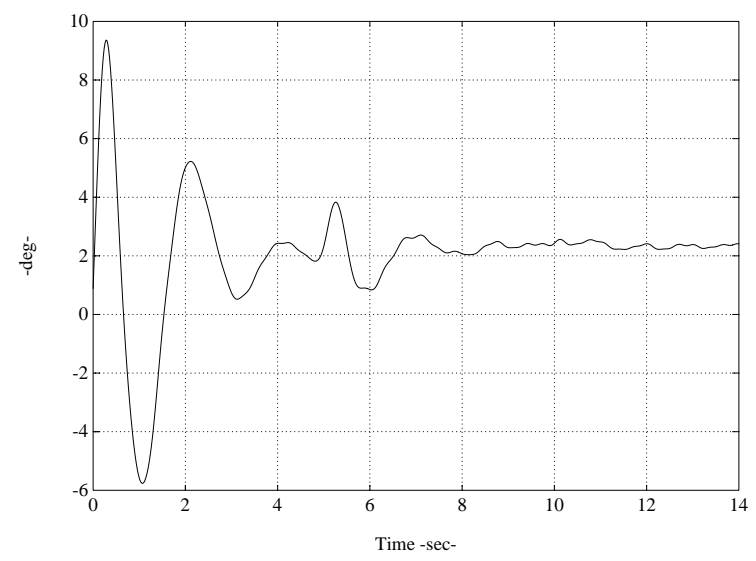

Fig. 16 - Tip deflection angle

In the second experiment, $\theta_{d}=(-3 \pi / 4,0)$ is the desired joint position to be reached from the same initial configuration, using as gains

$$
\widehat{K}_{P}=\operatorname{diag}\{5.7,6.2\}, \quad K_{D}=\operatorname{diag}\{2.5,1.34\} .
$$

The evolution of the error at joint 1 over 25 seconds is displayed in Fig. 17, showing that four updates are now necessary for obtaining convergence. No special care was taken for minimizing the duration of the motion: a faster global transient could have been obtained by updating sooner the feedforward $u_{2}$, then $u_{3}$, and finally $u_{4}$. This example shows the capability of learning the exact gravity compensation also when the 'wall' of maximum gravity force has to be overcome.

As a final experiment, the same motion was performed halving the positional gains in (40), i.e. with $\widehat{K}_{P}=\operatorname{diag}\{2.85,3.1\}$, satisfying hypothesis (b) but not the additional condition (c). Figure 18 shows 50 seconds of the first joint motion with no zeroing of the error (the two other joints have a similar behavior). Thus, relaxation of proportional gains by $50 \%$ will destroy the convergence guaranteed by our Theorem; this gives an upper bound for the distance to necessity of the stated conditions, also in the flexible case. 


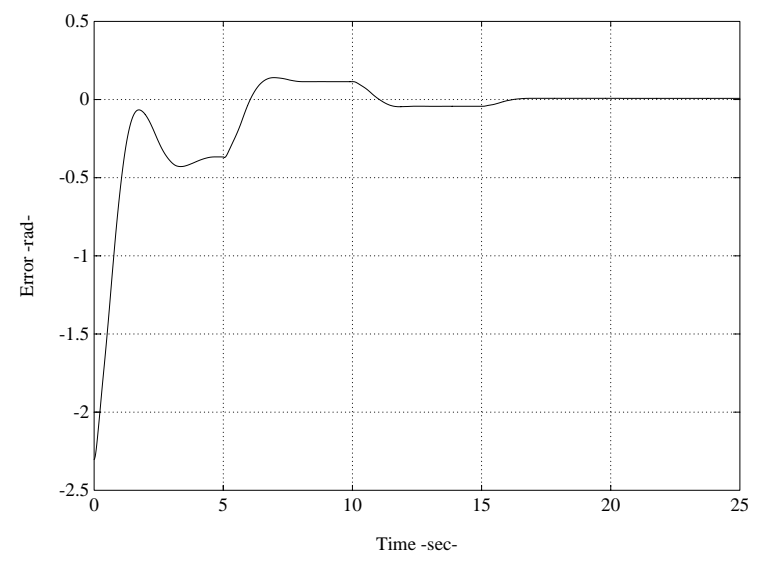

Fig. 17 - Position error for joint $1\left(\theta_{d}=(-3 \pi / 4,0)\right)$

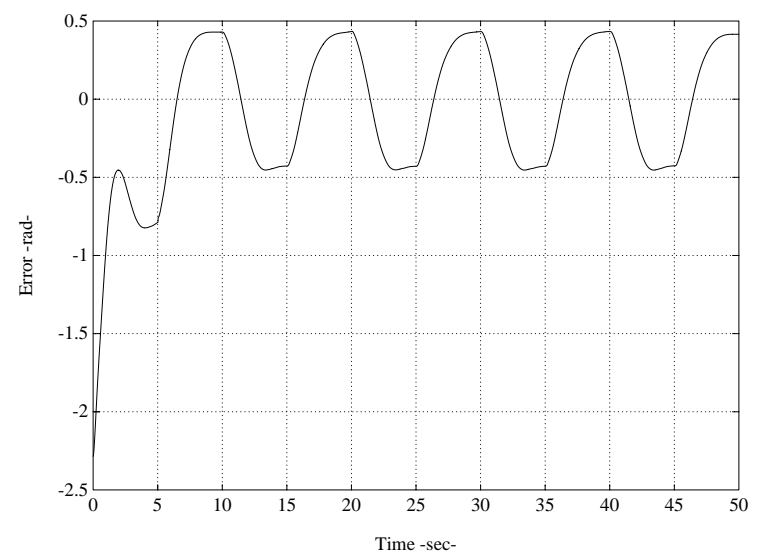

Fig. 18 - Position error for joint 1 with reduced gains

\section{CONCLUSIONS}

A simple iterative and model-independent control scheme has been presented for set-point regulation under gravity in a general class of robots, with or without flexibility concentrated at the joints or distributed along the links. The scheme generates the exact gravity compensation required at the set point, starting initially with a PD control law, and updating at discrete instants an additional feedforward term.

For all types of robot arms, the linear feedback is always closed at the motor level, thus using directly available variables. A lower bound condition on the magnitude of the proportional gain in the $\mathrm{PD}$ control part is sufficient to prove global convergence of the scheme. In the rigid case, this condition leads to a proportional gain which is twice as large as the one needed for ensuring the existence of a unique closed-loop equilibrium point. The same bound is obtained for the elastic joint case, while for robots with flexible links the 
bound is slightly increased. Both simulation and experimental results have shown the

effectiveness of the method, pointing out that the convergence conditions are also close to be necessary.

The approach was developed for the regulation of a desired configuration of the arm, as expressed by its motor (viz. joint) positions: deformation variables, when present, are not needed for feedback nor in the feedforward update. If the tip location of a flexible arm is of interest, a similar learning scheme can be derived, still closing the feedback loop at the motor level but taking into account the value of link (or joint) deformation in the process of updating the feedforward term at intermediate steady states.

\section{ACKNOWLEDGEMENTS}

This paper is based on work partly supported by Consiglio Nazionale delle Ricerche under contract 92.01115.PF67 (Progetto Finalizzato Robotica).

\section{REFERENCES}

[1] M. Takegaki and S. Arimoto, "A new feedback method for dynamic control of manipulators," Trans. ASME J. on Dynamic Systems, Measurement, and Control vol. 102, no. 2, pp. 119-125, 1981.

[2] P. Tomei, "A simple PD controller for robots with elastic joints," IEEE Trans. on Automatic Control, vol. 36, pp. 1208-1213, 1991.

[3] A. De Luca and B. Siciliano, "Relevance of dynamic models in analysis and synthesis of control laws for flexible manipulators," in Robotics and Flexible Manufacturing Systems, S.G. Tzafestas and J.C. Gentina (Eds.), Elsevier, Amsterdam, NL, pp. 161$168,1992$.

[4] H.G. Lee, S. Arimoto, and F. Miyazaki, "Liapunov stability analysis for PDS control of flexible multi-link manipulators," 27th IEEE Conf. on Decision and Control, Austin, TX, pp. 75-80, 1988.

[5] P. Tomei, "Adaptive PD controller for robot manipulators," IEEE Trans. on Robotics and Automation, vol. 7, pp. 565-570, 1991.

[6] A. De Luca and B. Siciliano, "An asymptotically stable joint PD controller for robot arms with flexible links under gravity," 31st IEEE Conf. on Decision and Control, Tucson, AZ, 1992.

[7] K.J. Åström and B. Wittenmark, Computer-Controlled Systems: Theory and Design, 2nd Ed., Prentice-Hall International, London, 1990.

[8] S. Arimoto and F. Miyazaki, "Stability and robustness of PID feedback control for robot manipulators of sensory capability," in Robotics Research: 1st Int. Symp., M. Brady and R.P. Paul (Eds.), MIT Press, Boston, MA, pp. 783-799, 1984. 
[9] F. Khorrami and Ü. Özgüner, "Decentralized control of robot manipulators via state and proportional-integral feedback," 1988 IEEE Int. Conf. on Robotics and Automation, Philadelphia, PA, pp. 1198-1203, 1988.

[10] A. De Luca, L. Lanari, P. Lucibello, S. Panzieri, and G. Ulivi, "Control experiments on a two-link robot with a flexible forearm," 29th IEEE Conf. on Decision and Control, Honolulu, HI, pp. 520-527, 1990.

[11] W.J. Book, "Recursive Lagrangian dynamics of flexible manipulator arms," Int. J. of Robotics Research, vol. 3, no. 3, pp. 87-101, 1984.

[12] A. De Luca and B. Siciliano, "Closed-form dynamic model of planar multi-link lightweight robots," IEEE Trans. on Systems, Man, and Cybernetics, vol. 21, pp. 826839, 1991.

[13] G.W. Stewart and J. Sun, Matrix Perturbation Theory, Academic Press, San Diego, 1990.

[14] A. De Luca and S. Panzieri, "A simple iterative scheme for learning gravity compensation in robot arms," 36th ANIPLA Annual Conf. (Automation 1992), Genova, I, pp. 459-471, 1992.

[15] A. De Luca and S. Panzieri, "An iterative scheme for learning gravity compensation in flexible robot arms," submitted to 12th IFAC World Congr., Sydney, AUS, Jul. 1993.

[16] S. Arimoto, S. Kawamura, and F. Miyazaki, "Bettering operation of robots by learning," J. of Robotic Systems, vol. 1, pp. 123-140, 1984.

[17] A. De Luca, G. Paesano, and G. Ulivi, "A frequency-domain approach to learning control: Implementation for a robot manipulator," IEEE Trans. on Industrial Electronics, vol. 39, pp. 1-10, 1992.

[18] M. Poloni and G. Ulivi, "Iterative learning control of a one-link flexible manipulator," 3rd IFAC Symp. on Robot Control, Vienna, A, pp. 393-398, 1991.

[19] A. De Luca and G. Ulivi, "Iterative learning control of robots with elastic joints," 1992 IEEE Int. Conf. on Robotics and Automation, Nice, F, pp. 1920-1926, 1992.

[20] P. Tomei, "An adaptive PD control algorithm for robots," in Robust Control of Linear Systems and Nonlinear Control, M.A. Kaashoek, J.H. van Schuppen, A.C.M. Ran Eds., Progress in Systems and Control Theory Series, vol. 4, pp. 575-582, Birkhäuser, Boston, 1990. 